\title{
Prenatal Toxicity of NNSS2 Iso- lated from Naja naja Shedded Skin in Pregnant Rats
}

\begin{abstract}
Keywords: NNSS2; Pregnancy; Prenatal toxicity; Snake shed skin
\section{Abstract}

Snake shed skin is a rich source of bioactive compounds possessing important therapeutic properties and is used extensively in folk and traditional medicine. From the Naja naja shedded skin an 882.6 Dalton peptide NNSS2 was isolated which caused cessation of the estrous cycle in female virgin mice. Although the role of NNSS2 in female virgin mice is well established but there is void regarding its role in pregnant animal, hence this study was undertaken to mechanistically understand the role of NNSS2 on pregnant rats.

In the present study NNSS2 altered urinary hydroxyproline, glucosamine at pre term and term during the gestational period. At term NNSS2 increased expression of serum estradiol, progesterone IL-1 $\beta$, TNF- $\beta$, and MMP-1 level where as it decreased serum IL-10 and Cathepsin - K level. In the post partum period NNSS2 decreased the expression of VEGF and MMP-2. Exposure of NNSS2 retarded the development process in the pups by altering the biomorphometric parameters.

Following NNSS2 exposure in the pregnant rats it was observed that it decreased liver and kidney organ weight in the pups. It increased liver (ALP, ASAT, ALAT) and kidney (ALP, urea, creatinine) enzymes markers. This study for the first time reports the toxicity of NNSS2 in pregnant rats and in the pups.
\end{abstract}

\section{Introduction}

Snakes shed skin although considered as a biological waste product is used in folk and ethno medicine of various cultures since ancient time without proper scientific validation. Recently Mukherjee et al., in 2013 for the first time established the role of Naja naja shedded skin in the estrous cycle [1]. The same group isolated a peptide namely NNSS2 from the Naja naja shedded skin and studied extensively its role on the estrous cycle of the female virgin mice. NNSS2 caused cessation of the estrous cycle for 5 days by decreasing serum progesterone, $\mathrm{P}_{4}-\mathrm{E}_{2}$ ratio and increasing IL-1 $\beta$ caspase 3,9 , Bax. NNSS2 also decreased decreased BCL2, HSP70, HSP90 [2]. Although the function of NNSS2 was well established in the estrous cycle but its role on pregnancy was not studied.

Pregnancy is a complex physiological phenomenon characterized by hemodynamic change together with anatomical and physiological changes in the urinary tract. Pregnancy remains under the influence of two major steroidal hormones; estrogen and progesterone, with increasing gestational age there is an increase in the level of estrogen and progesterone. Progesterone is mainly required for maintaining the quiescent endometrium preventing preterm delivery. Just prior to labour there is an increase in the level of estrogen 100-000 folds and in turn shifts the uterus from quiescent to rhythmically contractile state associated with an increase in production of pro inflammatory cytokines such as IL- $\beta$, IL-6, IL-8 and PGE2, which trigger cervical ripening $[3,4]$. The role of NNSS2 remain unexplored in pregnancy, hence this study for the first time tries to mechanistically explore its role in the female pregnant rats.

Journal of

Toxins

\author{
Mukherjee $\mathbf{S}^{1}$, Gomes $\mathrm{A}^{2}$ and Das Gupta SC ${ }^{1 *}$ \\ ${ }^{\prime}$ Department of Zoology Maulana Azad College, Rafi Ahmed Kidwai \\ Road, India \\ ${ }^{2}$ Department of Physiology, University of Calcutta, India \\ *Address for Correspondence \\ Das Gupta SC, Department of Zoology Maulana Azad College, 8, Rafi \\ Ahmed Kidwai Road, Kolkata 700013, India, Tel: 91-33-2226 0995; Fax-91- \\ 33--2226-0995; Mobile: + 91-9830471981; E mail: subirdgupta@gmail.com \\ Submission: 30 November, 2017 \\ Accepted: 28 December, 2017 \\ Published: 30 December, 2017 \\ Copyright: ๑ 2017 Mukherjee S, et al. This is an open access article dis- \\ tributed under the Creative Commons Attribution License, which permits \\ unrestricted use, distribution, and reproduction in any medium, provided \\ the original work is properly cited.
}

\section{Materials and Methods}

Chemicals and reagents-the following chemical and reagents were used on the course of the study: Acetyl acetone Himedia (India), Acrylamide E Merck (India), Albumin bovine serum Sigma (USA), RIPA lysis buffer Sigma (USA), Beta mercapto ethanol (BME) SRL (India), Bis acrylamide SRL (India), Bradford's reagent Amresco (USA), Ethanol E Merck (Germany), Nitro blue tetrazolium/ 5-bromo-4-chloro-3-indolyl phosphate (NBT-BCIP) Sigma (USA), Prestained molecular weight marker Fermentas (USA), Protease inhibitor cocktail (PIC) Amresco (USA), Pyrogallol SRL (India). Primary antibodies (MMP-2, VEGF) and corresponding secondary antibodies were purchased from Santa Cruz Inc. (USA).

The following kits were used for the present study: ALAT Ecoline Merck (India), ALP, ASAT, creatinine, were purchased from Ecoline Merck (India), Cathepsin-K and MMP-1 were purchased from Cusabio (China), 17- $\beta$ Estradiol $\left(\mathrm{E}_{2}\right)$, progesterone $\left(\mathrm{P}_{4}\right)$, IL-10, IL- $1 \beta$ and TNF- $\alpha$ were purchased from R\&D (USA).

Collection of N. naja shedded skin isolation and characterization of NNSS2- Fresh Naja naja shedded skins of both sexes were collected from North 24-Parganas, West Bengal, India through field collection as per the permission granted by the Ministry of Forests \& Wild Life, Govt. of West Bengal, India (2105/WL/4R-1 (PI-IX)) and were identified by the Zoological Survey of India. The shedded skins were store in desiccators at room temperature followed by aqueous extraction. The extract was subjected to thin layer chromatography, HPLC for purification to obtain active factor, and it was characterized through (MALDI, CD spectral analysis, H1NMR \& XRD) [2].

Animals and experimental design- Sexually mature male and female Wistar rats were obtained from the enlisted supplier of Maulana Azad College, Kolkata and allowed to acclimatize to laboratory conditions for at least 5 days prior to breeding. During breeding and until gestation day the rats were housed in individual cages. Animals were housed in such a way as to ensure uniform experimental conditions (ventilation and light) in rooms designed to maintain temperature at approximately $20-24{ }^{\circ} \mathrm{C}$. Relative humidity was maintained at $30-70 \%$, with a $12 \mathrm{hr}$ light-dark photo cycle. 
Virgin female rats (approximately120-130 g) were bred overnight with untreated adult males of the same strain (one male: two females) [5]. The day on which sperm were visualized in the vaginal smear was designated 0 day pregnant. Sperm-positive females were randomly assigned to test groups according to their day 0 of gestation. Feed and tap water were available adlibidum throughout the study. All experimental protocols described in this study were approved by institutional animal ethics committee and were in accordance with the guideline of the committee for the purpose of control and supervision of experiments on animal (CPCSEA), Government of India (Institutional Sanction No: 25/ 250/ 2012-AWD).

Treatment regime- Animals were randomized into 3 groups. Group 1 was non pregnant control, Group 2 was pregnant control and Group 3 was NNSS2 treated pregnant rats $\left(500 \mu . \mathrm{kg}^{-1}\right.$ body weight X 10 exposure, s.c). The drugs were administered from day 0 -day 9 of gestation.

Effect of NNSS2 on urinary biochemical markers- Urine was collected on day 14 (mid trimester period) and on day 20 (final trimester), urinary hydroxyproline and urinary glucosamine was assayed [6,7].

Effect of NNSS2 on urinary biochemical markers-On day 21 bloods was collected from the orbital plexus of the rats. The blood was kept at room temperature for $30 \mathrm{~min}$, and then placed at $4{ }^{\circ} \mathrm{C}$ for 30 $\mathrm{min}$, followed centrifugation at $5000 \mathrm{rpm}$ for $15 \mathrm{~min}$, and the serum was separated. From the serum hormones (E2, P4), cytokines (IL-1â, TNF-á, IL-10), Cathepsin-K, MMP-1 were assayed by Elisa (Biotek, USA; Model No. EL $\times 800$ MS) according to the manufacturers protocols. The concentrations of these hormones and cytokines were calculated from their respective standard curves.

Western immunoblot analysis - Expression of different proteins (VEGF, MMP-2 and $\beta$ - actin) of uterine tissue homogenate were analysed by western blot. Tissues were isolated from the pregnant female 30 days after parturition. 10\% tissue homogenate was prepared in RIPA lysis buffer along with protease inhibitor cocktail followed by centrifugation at $12,000 \mathrm{rpm}$ for $30 \mathrm{~min}$. The supernatant was collected and stored in $-20{ }^{\circ} \mathrm{C}$ for future use. Equal amount and volume of cellular protein samples were run in a $12.5 \%$ SDS PAGE and transferred to a nitrocellulose membrane in an immunoblot apparatus using transfer buffer (193 mM Glycine, $25 \mathrm{mM}$ Tris and $20 \%$ methanol) and were blocked using 5\% BSA in TBST for $2 \mathrm{~h}$. The membrane was next incubated with respective primary antibody diluted with TBST containing $0.3 \%$ BSA and kept for overnight. Next day, the membrane was washed with TBST to remove unbound primary antibodies and next incubated with respective secondary antibody (alkaline phosphatase conjugated). The membrane was washed with TBS and colour was developed using NBT-BCIP mix in dark. Finally membranes were scanned for future documentation. Each band was analyzed through Image J software for quantitative measurement.

Biomorphometry of pups- Gestational period of the pregnant female rats were monitored after prenatal exposure of NNSS2 (500 $\mu \mathrm{g} . \mathrm{kg}^{-1}$, body weight, $\mathrm{X} 10$ days, s.c) in pregnant rat. After 21 days of gestational period following birth of pups their biomorphometric features (body weight, head length, head diameter, neck with tail length) were monitored and measured with every 5 day interval using digital calliper (Mitutoyo, Japan) during the weaning period and continued till 30 days respectively.

Effect of NNSS2 on Liver and Kidney Metabolic Markers of pups- Following 1 month (PND 30 day) from the birth of pups they were sacrificed under anesthesia. Liver and kidneys were isolated and respective organ weights were measured. After that $10 \%$ liver and kidney homogenate was prepared in PBS ( $\mathrm{pH} 7.2$ ). With the tissue homogenate the following liver and kidney metabolic markers (ALAT, ASAT, ALP, Urea, and creatinine) were studied.

Statistical analysis- Statistical analyses were done by Graph Pad in Stat software (La Jolla, CA, USA). Data were expressed as mean \pm SEM $(n=4)$ unless otherwise mentioned. The differences between the treated and untreated control groups were analyzed by one-way ANOVA and post tests were done using Tukey's multiple comparison tests to determine the significant levels. ${ }^{*} \mathrm{p}<0.05,{ }^{* *} \mathrm{p}<$ $0.01,{ }^{* * *} \mathrm{p}<0.001$ were considered significant.

\section{Results}

Effect of NNSS2 on urinary biochemical markers - NNSS2 (500 $\mu \mathrm{g} . \mathrm{kg}^{-1}$ body weight, X 10 days, s.c) (day 0-day 9 of gestation period) treatment in pregnant animal produced significant decrease in urinary hydroxyproline level on day 14 and significant increase in urinary hydroxyproline on day 20 of gestation as compared with pregnant control rat. NNSS2 produced significant decrease in urinary glucosamine level on day 14 of gestation, but did not produce significant change in urinary glucosamine on day 20 of gestation as compared with pregnant control rat (Figure 1A, 1B).

Effect of NNSS2 on serum biochemical markers - NNSS2 (500 $\mu . \mathrm{kg}^{-1}$ body weight, X 10 days, s.c) (day 0 -day 9 of gestation period) treatment in pregnant animal produced significant increase in the serum $\mathrm{E}_{2}$ and $\mathrm{P}_{4}$ level on day 21 of gestation as compared with pregnant control (Figure 2). NNSS2 produced significant decrease in the serum IL-1 $\beta$ on day 21 and significant increase in serum TNF-á and serum IL-10 on day 21 of gestation as compared with pregnant control rat (Figure 3 ). NNSS2 produced significant increase in serum MMP-1 and decrease in serum Cathepsin-K on day 21 of gestation as compared with pregnant control (Figures 4, Figure 5).

Effect NNSS2 on VEGF and MMP-2 expression-NNSS2 (500 $\mu . \mathrm{kg}^{-1}$ body weight, $\mathrm{X} 10$ days, s.c) (day 0-day 9 of gestation) treatment in pregnant animal caused significant decrease in uterine tissue VEGF and MMP-2 expression 30 days after parturition as compared with pregnant control. Quantitative analysis through Image J software also confirmed the down regulation of uterine tissue VEGF and MMP-2 expression as compared with pregnant control (Figure 6A and 6B).

Effect of NNSS2 on biomorphometry and physiological parameters of the pups- NNSS2 caused significant increase in body weight on day 0 up to day 10 as compared with control pups but produced significant decrease in body weight from day 15 up to day 30 as compared with control pups (Figure 7A). NNSS2 did not produce significant change in head length on day 0 as compared with control pups but significant decrease was observed in head length on day 5 up to day 30 (Figure 7B). NNSS2 did not produce significant decrease in head diameter on day 0 up to day 10 as compared but decrease in head diameter was observed on day 15 up to day 30 (Figure 7C). NNSS2 caused significant decrease in neck width on day 

6.

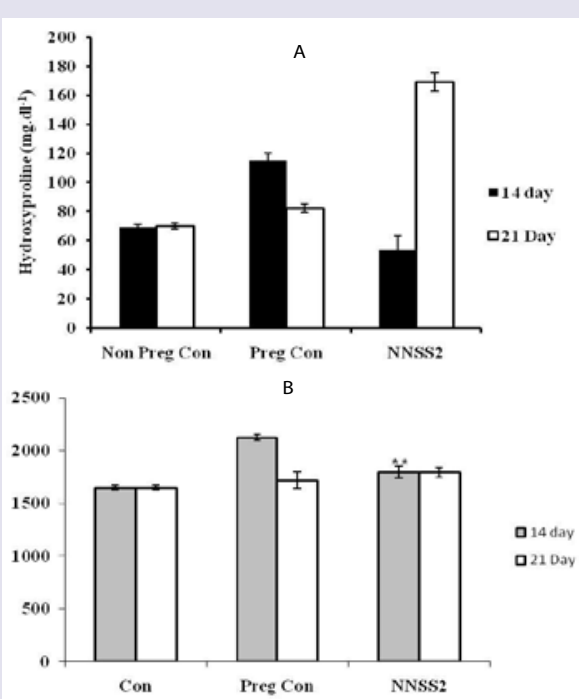

Figure 1: Effect of NNSS2 on urinary hydroxyproline and glucosamine. 0 day pregnant rats were treated with NNSS2 $\left(500 \mu \mathrm{g} \cdot \mathrm{kg}^{-1}\right.$ body weight, $X 10$ days, s.c) (day 0 -day 9 of gestation period).

(A) NNSS2 produced significant decrease in urinary hydroxyproline on day 14 and increase on day 21 of gestation as compared with pregnant control rat. (B) NNSS2 produced significant decrease in urinary glucosamine on day 14 of gestation, but did not produce significant change on day 20 of gestation period as compared with pregnant control rat.

Values were represented as mean \pm SEM ${ }^{* *} p<0.01{ }^{* * *} p<0.001$

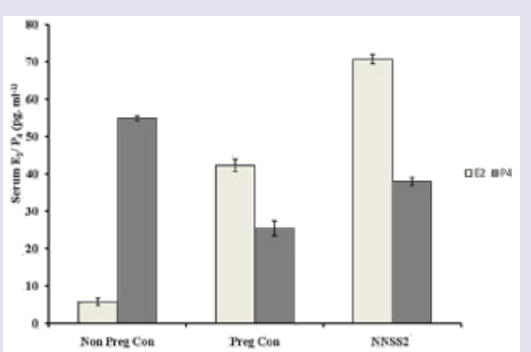

Figure 2: Effect of NNSS2 on serum Estradiol $\left(E_{2}\right)$ and progesterone $\left(P_{4}\right)$. 0 day pregnant rats were treated with NNSS2 (500 $\mu \mathrm{g} . \mathrm{kg}-1$ body weight, $X$ 10 days, s.c) (day 0 -day 9 of gestation period). NNSS2 produced significant increase in serum $\mathrm{E}^{2}$ and serum $\mathrm{P}^{4}$ on day 21 of gestation as compared with pregnant control rat.

Values were represented as mean \pm SEM $(n=4) .{ }^{* *} p<0.01,{ }^{* * *} p<0.001$ (Significant).

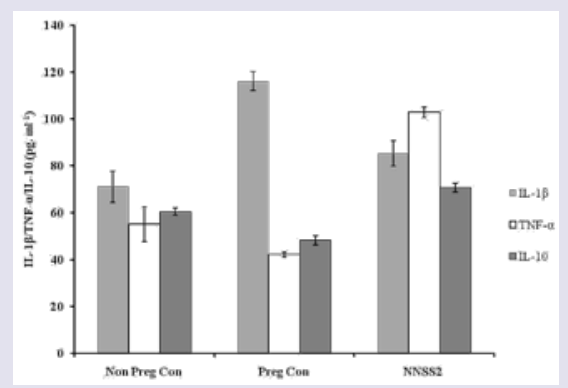

Figure 3: Effect of NNSS2 on serum cytokines (IL-1 $\beta$ TNF- $\beta$ and IL-10) 0 day pregnant rats were treated with NNSS2 (500 $\mu$ g.kg- 1 body weight, $X$ 10 days, s.c) (day 0 -day 9 of gestation period). NNSS2 (500 $\mathrm{gg}_{\mathrm{kg}} \mathrm{kg}^{-1}$ body weight, $X 10$ days, s.c) produced significant decrease in serum IL $-1 \beta$, significant increase in TNF- $\alpha$ and IL-10 on day 21 of gestation as compared with pregnant control rat.

Values were represented as mean \pm SEM $(n=4)$. ${ }^{*} p<0.05,{ }^{* *} p<0.01,{ }^{* * *}$ $p<0.001$ (Significant)

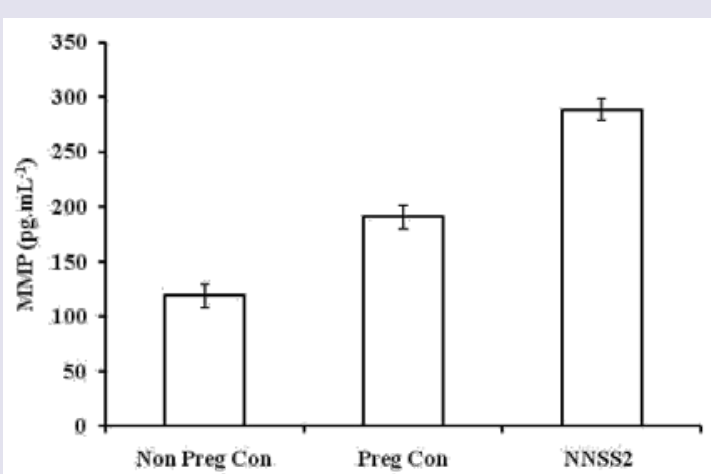

Figure 4: Effect of NNSS2 on serum MMP-1.

0 day pregnant rats were treated with NNSS2 (500 $\mu \mathrm{g}^{\mathrm{kg}} \mathrm{kg}^{-1}$ body weight, $\mathrm{X}$ 10 days, s.c) (day 0 -day 9 of gestation period). NNSS2 produced significant increase in serum MMP-1 on day 21 of gestation as compared with pregnant control animal.

Values were represented as mean \pm SEM $(n=4) .{ }^{* *} p<0.01$ (Significant).

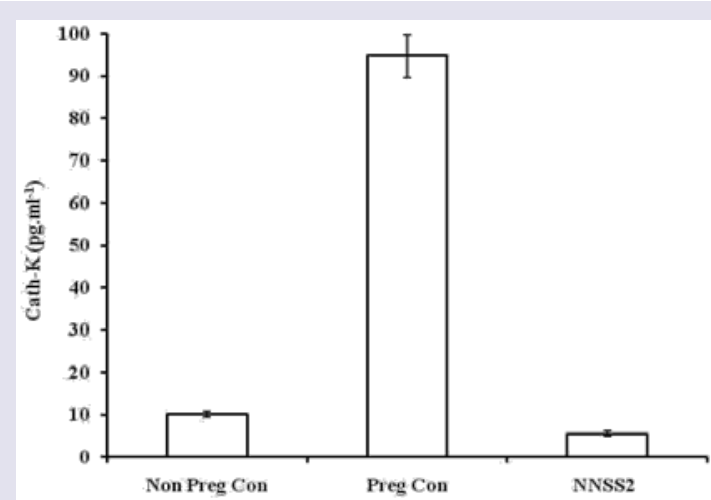

Figure 5: Effect of NNSS2 on serum Cathepsin-K.

0 day pregnant rats were treated with NNSS2 $\left(500 \mu \mathrm{g}^{\mathrm{kg}}{ }^{-1}\right.$ body weight, $X$ 10 days, s.c) (day 0 -day 9 of gestation period). NNSS2 produced significant decrease in serum Cath-K on day 21 of gestation as compared with pregnant control rat.

Values were represented as mean $\pm \operatorname{SEM}(n=4) .{ }^{* * *} p<0.001$ (Significant).

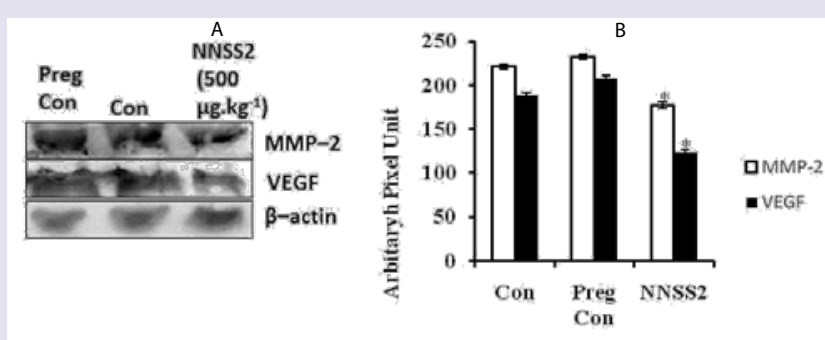

Figure 6: Effect of NNSS2 on uterine VEGF and MMP-2 after 1 month followed parturition.

(A) NNSS2 (500 $\mu$ g.kg-1 body weight, $X 10$ days, s.c) treated uterine tissue Protein from the total tissue lysate was subjected to SDS-PAGE and western immunoblot analysis was done using VEGF, MMP-2 and â-actin primary antibody. After primary antibody incubation, respective secondary antibodies were added and colour development was performed using NBT/ BCIP mix. The relative intensity of each band was measured after normalization with the intensity of â-actin in a blot (shown below each western blot). Representative blots from three independent experiments gave identical results.

(B) NNSS2 (500 $\mu \mathrm{g} \mathrm{kg}^{-1}$ body weight, $X 10$ days, s.c) treated uterine tissue. After western blot analysis densitometry analysis of the respective protein bands were performed. Histograms showed representative pixel intensities (arbitrary units of densitometry analysis using Image $\mathrm{J}$ software) of the immunoblot. Values were represented as mean \pm SEM. 
5 up to day 30 (Figure 7D). NNSS2 did not produce significant change in tail length on day 0 up to day 10 as compared with control pups. NNSS2 caused significant decrease in tail length from day 15 up to 30 (Figure 7E).

Effect of NNSS2 Liver and Kidney Metabolic Markers of pupsNNSS2 significantly decrease liver and kidney weight as compared with control pups (Figure 8A). NNSS2 produced significant increase in the level of liver ALP ALAT and ASAT activity as compared with control pups (Figure 8B). NNSS2 produced significant decrease in kidney weight as compared with control pups. NNSS2 produced significant increase the level of kidney ALP urea and creatinine as compared with control pups (Figure 8C).

\section{Discussion}

Pregnancy is one of the major events in female reproductive physiology and involves several biochemical and physiochemical events. There occur molecular cross talks between mother and fetus which are governed by various factors where progesterone and estrogen played key regulatory role, and are mediated by cytokines, growth factors inducing extensive tissue remodelling $[5,8]$.

Glucosamine is formed in the body as glucosamine 6-phosphate (G6-P), it is the most fundamental building block essential for the biosynthesis glycolipids, glycoproteins, glycosaminoglycans hyaluronate and proteoglycans, requiring amino sugars. Glucosamine

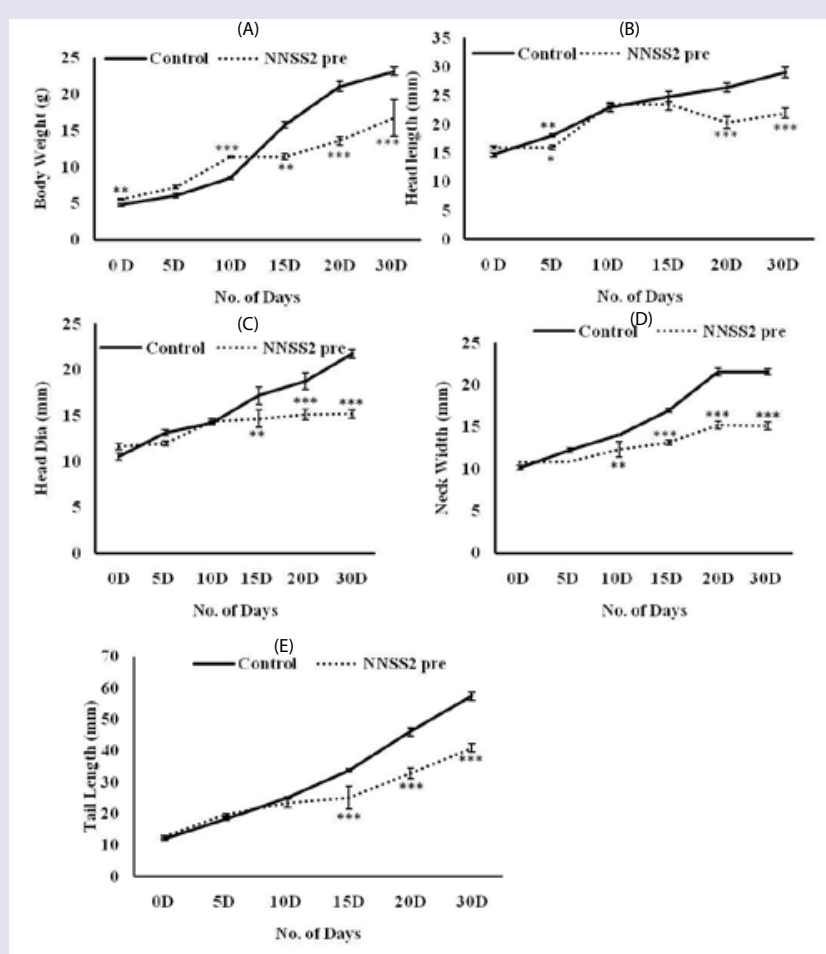

Figure 7: Effect of NNSS2 on pup biomorphometry following prenata exposure 0 day pregnant rats were treated with NNSS2 pre $\left(500 \mu \mathrm{g}^{\mathrm{kg}}{ }^{-1}\right.$ body weight, $X 10$ days, s.c) (day 0 -day 9 of gestation period). 0 day lactating animal were treated with NNSS2 post $(500 \mu \mathrm{g} . \mathrm{kg}-1$ body weight, $X 10$ days, s.c) (PND 0-PND 9).

(A) Body weight in g; (B) Head length in $\mathrm{mm}$; (C) Head diameter in $\mathrm{mm}$; (D) Neck width in $\mathrm{mm}$; (E) Tail length in $\mathrm{mm}$.

Values were represented as mean \pm SEM. ${ }^{*} p<0.05,{ }^{* *} p<0.01,{ }^{* * *} p<0.001$ (Significant).

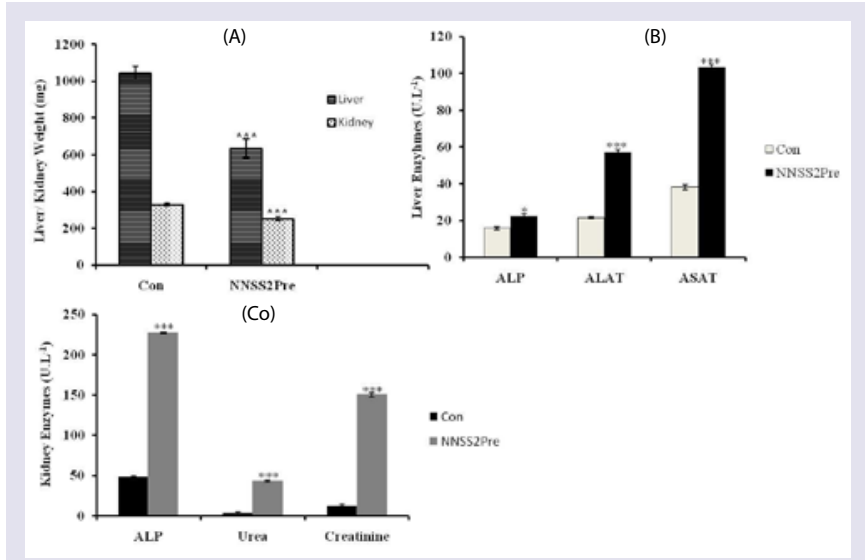

Figure 8: Effect of NNSS2 Liver and Kidney Metabolic Markers of pups.

(A) 0 day pregnant rats were treated with NNSS2 $\left(500 \mu \mathrm{g} \cdot \mathrm{kg}^{-1}\right.$ body weight, $X$ 10 days, s.c) (day 0-day 9 of gestation period). NNSS2 produced significant decrease in kidney weight as compared with control pups.

(B) NNSS2 produced significant increase in liver ALP as compared with control pups.

(C) NNSS2 produced significant increase in kidney ALP as compared with control pups. NNSS2pre produced significant increase in kidney urea and creatinine as compared with control pups.

Values are mean \pm SEM. ${ }^{* *} p<0.01,{ }^{* *} p<0.001$ (Significant)

is the primary amino sugar substrate for the biosynthesis of macromolecules, such as chondroitin sulphate and hyaluronic acid, which provided the backbone for collagen formation [9]. Hydroxyproline (HP) is a modified amino acid that is derived from proline by post-translational hydroxylation. HP is liberated following collagen breakdown. Out of the total HP released, $90 \%$ of it is degraded into free amino acid, which is further catabolized into urea and $\mathrm{CO}_{2}$; and remaining $10 \%$ are excreted in the urine. The $\mathrm{C} 1 \mathrm{q}$ fraction of complement contained a structural region that is similar to collagen and alteration or increased inflammatory response is associated with increase HP release and excretion. The other factors altering the HP release is shifting of the ratio between bone formation and bone resorption, and $\mathrm{HP}$ acts as a marker of bone mass transfer and break down [10].

NNSS2 (500 $\mu$ g.kg-1 body weight, X 10 days, s.c) (day 0-day 9 of gestation period) treatment in pregnant decreased urinary excretion of HP during mid trimester (day 14), and increased during the last trimester (day 20), glucosamine was decreased during mid trimester, suggested that during the mid trimester there is increased chances of maternal osteomalacia. At the last trimester of pregnancy increased excretion of HP suggested increased collagen breakdown increased inflammatory response (Figure 1A and Figure 1B).

The uterus must remain fairly quiescent state during gestation leading to the normal growth and development of the feto-placental unit, after completion of this prior to parturition the uterus transformed into a strongly contractile structure. This is associated with changes in the density and activity of ion channels and pumps, and formation of gap junctions, which facilitated the spread of activity throughout the muscle cells in the uterine wall. Labour induction leads to an increase in estrogen and decrease in maternal progesterone. Activation is associated with increased expression of the gap junction protein, connexion-43 (Cx-43), oxytocin receptor and prostaglandin receptor. Expression of these contraction associated proteins (CAPs) 
was regulated by increasing estrogen and decreasing progesterone.

NNSS2 increased both estrogen and progesterone expression indicated higher recruitment of CAP proteins, oxytocin receptors and prostaglandin expressions but on the other hand increased progesterone. Therefore NNSS2 altered normal endocrine milieu during the onset of labour induction (Figure 2 and Figure 3).

Pro inflammatory cytokines played a critical role in normal (term) and abnormal (preterm) pregnancy. IL- $1 \beta$, TNF- $\alpha$ is associated with number of cellular responses and regulated both cellular survival and apoptosis. These cytokines are mainly secreted by the placenta and the gestational tissues. Other than the pro inflammatory cytokines like IL-1 $\alpha$, TNF- $\alpha$ gestational tissue also secrete anti inflammatory cytokines like IL-10 which in fact stimulated the release of TNF- $\alpha$. NNSS2 treatment in pregnant animal produced increased expressions of TNF- $\alpha$, IL- $1 \beta$ and IL-10 expression leading intrauterine growth restriction of the pups (Figure 3).

During parturition adult tissue undergo rapid tissue remodelling associated with rapid changes in tissue mass and function. MMP's are secreted as proenzyme and are secreted into the extracellular matrix, and their concentration and activity are regulated by several endocrine factors and cytokines. Cathepsin $\mathrm{K}$ is a protease with high collagenolytic and elastinolytic activity and its cellular expression was previously thought to be restricted to osteoclasts and osteoclastmediated bone resorption only [11-13]. Recent studies had suggested the presence of cathepsin $\mathrm{K}$ in the embryo and fetus and play a major role in the degradation of extracellular structure proteins and basement membrane components [14]. NNSS2 caused an increase expression of MMP-1 and decreased Cathepsin $\mathrm{K}$ when compared with pregnant control (Figure 4 and 5).

From the NNSS2 treated pregnant animal uterus was collected after 1 month following parturition. From the tissue homogenate expression of VEGF and MMP-2 were studied. NNSS2 significantly decreased uterine expression of MMP-2 and VEGF as compared with pregnant control. In the uterus MMP-2 gets expressed in the uterine stromal cell and degraded the collagen bundles both type I and type IV. On the other hand, VEGF played critical role post partum repair and healing and recovery of the cervix to a non-pregnant state (Figure $6 \mathrm{~A})$.

When the corresponding biomorphometric changes were studied it was seen prenatal maternal exposure of NNSS2 caused significant decrease in body weight, head length, head diameter, tail length and neck width, suggesting role in impediment of growth and development of pups (Figure 7).

Liver is the main metabolic house so expressions of hepatotoxic markers were studied. Expressions of the following hepatotoxic markers ASAT (Aspartate amino transferase), ALAT (Alanine amino transferase), ALP (Alkaline phosphatase) were studied from the liver tissue homogenate. NNSS2 increased the expression of ASAT, ALAT, ALP when compared with control untreated pups. ASAT, ALAT are the major hepatotoxic markers released due to severe membrane damage, disintegration, cellular level injury and necrosis and hepatocellular inflammation which ultimately leads to loss of functional integrity. ALP was released due to membrane damage and loss of function of hepatocytes [15]. Elevation of these enzymes directed altered hepatacellular membrane permeability; they are released through basal-lateral side of hepatocytes to facing the sinusoids due to metabolic stress. Therefore it could be concluded that NNSS2 caused metabolic stress leading to hepatic damage (Figure 8).

NNSS2 (prenatal exposure) caused renal dysfunction as was evident from the increased tissue level expression nephrotoxic biomarker enzymes (ALP) and nitrogeneous end products (creatinine, urea). The increase in ALP activity is due to the injury to the brush border membrane of the renal tubular cells [16]. Creatinine is a bio marker for renal tubular lesion which reflects on loss of glomerular filtration function accompanying the onset of nephrotoxicity. An increase in the urea is a direct marker for nephropathy and brings about local kidney inflammatory responses (Figure 8).

NNSS2 hindered the developmental process by altering the maternal biochemical profile as were also evident from the biomorphometric changes of pups. All the above factors indicated that NNSS2 is associated with impairment bone tissue development; hence had a crucial role in inhibiting the normal developmental process of fetus and suggesting fetal growth retardation. So it was apparent that being a small molecule during prenatal exposure it passed through the feto-placental barrier and then altered the metabolic status of the pups.

\section{References}

1. Mukherjee S, Dasgupta SC, Gomes A (2013) Effect of Naja naja Laurenti shed skin extract on estrous cycle, hormone - cytokine profiles, histopathology of ovary and uterus of Swiss albino mice. Indian J Exp Biol 51: 235-240.

2. Mukherjee S, Dasgupta SC, Gomes A (2016) Estrous cycle cessation caused via activating the apoptotic pathway by NNSS2 Isolated from Naja naja shedded skin. J Toxins 3: 1-5.

3. Hendrick V, Altshuler LL, Suri R (1998) Hormonal changes in the postpartum and implications for postpartum depression. Psychosomatics 39: 93-101.

4. Konopka CK, Morais EN, Naidon D, Pereira AM, Rubin MA, et al. (2013) Maternal serum progesterone, estradiol and estriol levels in successful dinoprostone- induced labor. Braz J Med Biol Res 46: 91-97.

5. Hulboy DL, Rudolph LA, Matrisian LM (1997) Matrix metalloproteinases as mediators of reproductive function. Mol Hum Reprod 3: 27-45.

6. Neuman RE, Logan MA (1950) The determination of hydroxyproline. J Biol Chem 184: 299-306.

7. Elson LA, Morgan WT (1933) A colorimetric method for the determination of glucosamine and chondrosamine. Biochem J 27: 1824-1828.

8. Cha J, Sun X, Dey SK (2012) Mechanisms of implantation: strategies for successful pregnancy. Nat Med 18: 1754-1767.

9. Kelly GS (1998) The role of glucosamine sulfate and chondroitin sulfates in the treatment of degenerative joint disease. Altern Med Rev 3: 27-39.

10. Simsek B, Karacear O, Kara I (2004) Urine products of bone breakdown as markers of bone resorption and clinical usefulness of urinary hydroxyproline: an overview. Chin Med J (Engl) 117: 291-295.

11. Bromme D, Okamoto K, Wang BB, Biroc S (1996) Human cathepsin O2, a matrix protein-degrading cysteine protease expressed in osteoclasts. Functional expression of human cathepsin $\mathrm{O} 2$ in Spodoptera frugiperda and characterization of the enzyme. J Biol Chem 271: 2126-2132.

12. Bossard MJ, Tomaszek TA, Thompson SK, Amegadzie BY, Hanning CR, et 
al. (1996) Proteolytic activity of human osteoclast cathepsin K. Expression, purification, activation, and substrate identification. J Biol Chem 271: 1251712524.

13. McQueney MS, Amegadzie BY, D'Alessio KD, Hanning CR, McLaughlin MM, McNulti et al. (1997) Autocatalytic activation of human cathepsin K. J Biol Chem 272: 13955-13960.

14. Haeckel C, Krueger S, Buehling F, Broemme D, Franke K, et al. (1999)
Expression of cathepsin $\mathrm{K}$ in the human embryo and fetus. Dev Dyn 216: 89-95.

15. Sahreen S, Khan MR, Khan RA (2011) Hepatoprotective effects of methanol extract of Carissa opaca leaves on $\mathrm{CCl} 4$-induced damage in rat. BMC Complement Altern Med 11: 48.

16. Leibovitch I, Ben-Chaim J, Ramon J, Goldwasser B (1991) Increased serum alkaline phosphatase activity: a possible indicator of renal damage. J Clin Lab Anal 5: 406-409.

\section{Acknowledgements}

We thankfully acknowledge University Grant Commission, New Delhi, India for partial financial assistance (Ref No: F 38-130 (SR), dated 19.12.2009 and we are thankful to the Dept. of Forests \& Wild Life, Govt. of West Bengal, India (2105/WL/4R-1(PI- IX)) for kind permission of collection of the shed snake skin. 